\title{
Two-dimensional Model of Self-Pulsation in AlGaAs Laser Diodes
}

\author{
Canice O'Brien $^{\mathrm{a}}$, Woon-Ho Seo ${ }^{\mathrm{b}}$, Heidi K. Thornquist ${ }^{\mathrm{c}}{ }^{\uparrow}$ and John F. Donegan ${ }^{\mathrm{a} *}$ \\ ${ }^{\mathrm{a} S e m i c o n d u c t o r ~ P h o t o n i c s ~ G r o u p, ~ D e p a r t m e n t ~ o f ~ P h y s i c s, ~ T r i n i t y ~ C o l l e g e ~ D u b l i n, ~ D u b l i n ~ 2, ~ I r e l a n d, ~}$ \\ ${ }^{\mathrm{b}}$ Department of Electronic and Electrical Engineering, Sungkyunkwan University, 440-746, Korea, \\ ${ }^{\mathrm{c}}$ Sandia National Laboratories, P.O. Box 5800, MS 1110 \\ Albuquerque, NM 87185-1110, U.S.A.
}

\begin{abstract}
The use of a fully two-dimensional waveguide analysis of the optical modes in a self-pulsating laser is described. We show that there is a need for such an analysis as the effective index model often used for modeling semiconductor lasers is shown not to provide a correct optical mode when the lateral waveguide is only weakly guiding. An efficient technique for solving the large and sparse matrix calculation in the two-dimensional model, the implicitly restarted Arnoldi method, is used and shown to be useful for the repetitive calculation necessary to describe the laser dynamics. We find that the optical field and optical gain show a variation in the pulse cycle and that this variation that occurs due to the change in carrier density, is responsible for the development of self-pulsation in the diode device.
\end{abstract}

Key-words: AlGaAs materials, self-pulsating lasers, optical waveguide analysis.

\section{INTRODUCTION AND BACKGROUND}

Self-pulsating lasers are a class of laser that emit short optical pulses of 1-2 ps duration at GHz repetition rates ${ }^{1}$. The AlGaAs material system with an active region at $780 \mathrm{~nm}$ is the best studied system ${ }^{2}$. Newer materials systems in the red and blue spectral regions have also been proposed and developed ${ }^{3,4}$. As these lasers are pulsed sources, they are relatively immune to the effects of optical feedback and have found use in readout systems in compact-disc players. The basic structure of the laser is given in Fig. 1. We see an active layer region above which there are current blocking and current spreading layers. The combination of stripe width, current blocking and current spreading widths determines the current profile in the device. As there is no material variation in the lateral direction, the optical mode is only weakly confined. The different material compositions in the current blocking and the cladding layers result in a small effective index step in the lateral direction under the current stripe. The addition of carriers alters this step refractive index profile and results in the spreading of the lateral mode. It is the spreading of this mode that causes the laser to cease operation and thereby to emit a pulse. The effect is repeated and a train of pulses is generated in the laser. It is possible to model such a laser in the effective index model where the modes in the transverse direction and the lateral direction are determined separately, this is termed a $2 \times 1 \mathrm{D}$ effective index model calculation ${ }^{5}$. In this paper, we demonstrate the importance of a fully two-dimensional calculation of the optical field within the waveguide of a self-pulsating laser based in the AlGaAs material system. We demonstrate the weaknesses of the effective index method for structures incorporating weak lateral guiding. It is shown that in order to achieve the solution of the two-dimensional system, the numerical solution of the non-hermitian eigenvalue problem is necessary. Based on the use of the implicitly restarted Arnoldi method and taking advantage of the inherent symmetry within the device, the two dimensional field profiles are calculated in a computationally efficient manner using a finite difference scheme. This allows us to understand the mechanism of self-pulsation based on the results of the model. We find that the width of the optical field oscillates and shows a variation of 0.45 microns over the pulse period. The gain also oscillates and this results in laser action that is pulsed when the gain exceeds all losses while the lasing action is quenched when the losses are too large.

*jdonegan@tcd.ie; phone 353-1-6081987; fax 353-1-6711759

${ }^{\uparrow}$ Sandia is a multiprogram laboratory operated by Sandia Corporation, a Lockheed-Martin Company, for the United States Department of Energy under Contract DE-AC04-94AL85000 


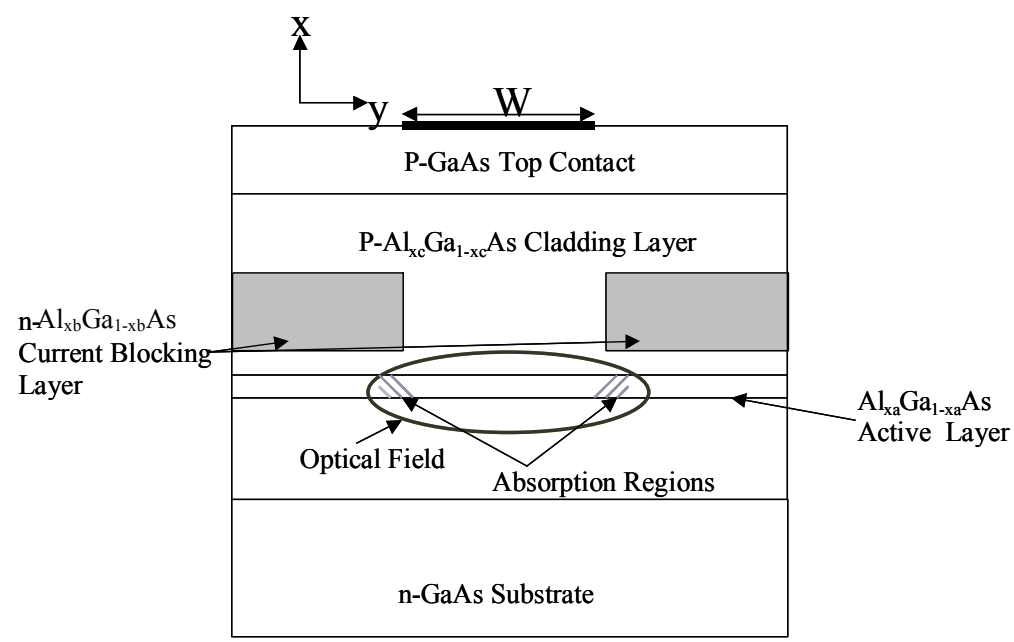

Figure 1. Schematic diagram of the self-pulsating device. The optical field is indicated and is only weakly guided in the lateral direction.

\section{EFFECTIVE INDEX METHOD}

The effective index method is an approximate technique for solving the two-dimensional given by equation (1) by separation of variables. Provided the lateral (y direction in Fig. 1) refractive index variation is much less than the transverse index variation ( $\mathrm{x}$ direction in Fig.1 $)$ then we can approximate the two-dimensional electric field as:

$$
E(x, y)=\psi\left(x, y_{i}\right) \Phi(y)
$$

where $\psi\left(x, y_{i}\right)$ is the transverse electric field in the $\mathrm{x}$ direction at the point $y_{i}$. The first step of the effective index technique is to find the effective index values at each point $y_{i}$ for all of the lateral sections. The effective index is simply defined as the transverse propagation constant divided by the vacuum wavenumber:

$$
n_{\text {eff }}\left(y_{i}\right)=\frac{\beta_{x}}{k_{0}}
$$

Then the one dimensional slab waveguide comprising the effective index for each of the lateral sections is solved. Unfortunately although the effective index is very accurate for strongly guided waveguides, the technique becomes unreliable as the fundamental mode approaches cutoff. When the waveguide is close to anti-guiding, the lateral profile develops a very different profile and in particular the mode has significant intensity far away from the stripe. This would in turn greatly affects the operation of the laser structure within the model. For our modeling, we chose an Aluminium content $\mathrm{x}_{\mathrm{a}}$ of 0.14 in the active region, an $\mathrm{x}_{\mathrm{b}}$ of 0.55 in the blocking layer and an $\mathrm{x}_{\mathrm{c}}$ of 0.5 in the cladding layer. For these parameters, the effective index step is $3.7 \times 10^{-3}$. The rate of change of the refractive index with carrier density is denoted by $\frac{d n}{d N}=-1.2 \times 10^{-26} \mathrm{~m}^{-3}$. . The calculation of the optical confinement factor for the guide is shown in Fig. 2. We see that the mode is no longer the single-peaked mode that would be expected. The material parameters were chosen so that at carrier densities about $1 \times 10^{24} \mathrm{~m}^{-3}$ in the lasing regime, the guide would be above cutoff. As the selfpulsation process depends critically on the shape of the optical mode in particular its extent in the lateral direction, the mode calculated in this effective index model will not properly describe the self-pulsation behaviour. 


\section{TWO-DIMENSIONAL WAVEGUIDING}

The need for a full two-dimensional solution of the wave equation is clear from Fig.2. The side lobes that appear close to anti-guiding within the effective index approximation result in an overestimate of the modal absorption within the regions of the active layer laying beneath the current stripe. It was therefore necessary to develop a simulator based on the two-dimensional finite difference method for accurate modeling of the self-pulsation process.

The task involved in solving the resulting $2 \mathrm{D}$ matrix is very large indeed as the computation effort scales approximately with the cube of the order of the matrix to be solved ${ }^{7}$. The first step was to use the inherent symmetry of the waveguide in the lateral direction so that it was only necessary to solve for half of the waveguide with a subsequent reduction by a factor of 8 in the computational time. The matrix created from the guide geometry has an order of 3060 but has only $0.16 \%$ of its elements as non-zero. A matrix solver for sparse non-Hermitian matrices was therefore sought. We impose the condition

$$
\operatorname{Re}\left(n_{\text {Clad }}\right) \leq \operatorname{Re}\left(\beta_{z}\right) \leq \operatorname{Re}\left(n_{\text {Well }}\right)
$$

as only the bound modes need to be calculated. The largest modal gain so that

$$
\operatorname{Max} \operatorname{Im}\left(\beta_{z}\right)>0
$$

is chosen since only the lasing mode needs to be considered here. The saving in computational effort by choosing an algorithm that computes this very limited subset of eigenpairs, is very considerable. In order to model the laser dynamics, the matrix calculation must be carried out for each $1 \mathrm{ps}$ time interval over a $5 \mathrm{~ns}$ time duration, and so a very efficient means of solving for the eigenvectors of our sparse matrix is needed.

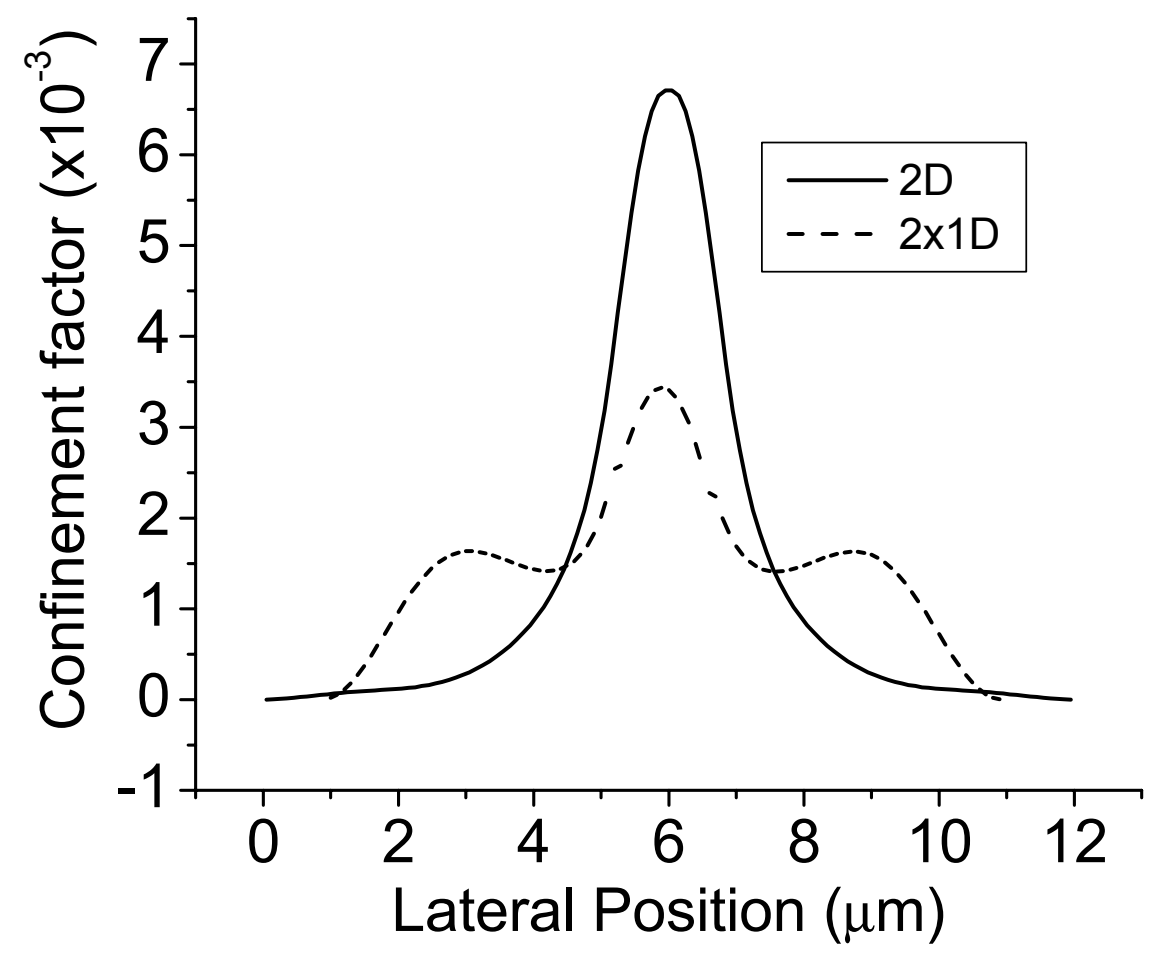

Figure 2. The optical confinement factor for both the $2 \mathrm{D}$ and $2 \times 1 \mathrm{D}$ calculations. It is clear that the mode is properly calculated only in the $2 \mathrm{D}$ calculation. 
We use the Arnoldi method for the computation of the eigenvectors and the eigenvalues using a finite difference method 8,9 . The technique is helped by the use of eigenvector information from the previous results to compute the results of the present matrix. The time taken for a standard simulation of 5000 points over the 5 ns time scale was 21 hours and 12 minutes on a $2.2 \mathrm{GHz}, 256 \mathrm{MB}$ RAM personal computer. This period while long, is much less than would be found if it were necessary to use a solver that evaluated all the eigenvectors and eigenvalues of a dense large matrix.

\section{TWO DIMENSIONAL FIELD PROFILE}

The calculated field profile for the 2D case at the peak modal point is shown in Fig.3 The peak of the intensity profile coincides with the position of the active layer as shown in Fig. 1. It is clear from this profile that through the correct application of the symmetry line boundary conditions, the field profile behaves as though the whole waveguide were present. This allows for a much shorter computation time as outlined above.

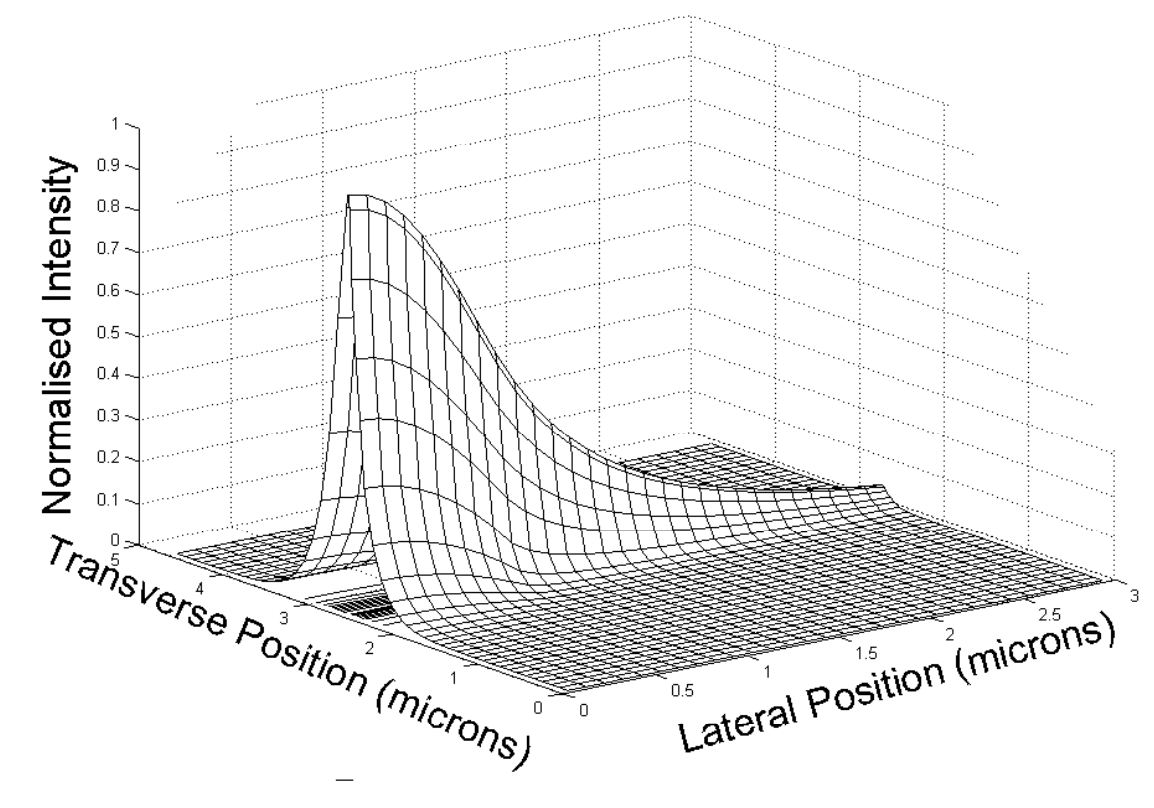

Figure 3 Normalised intensity distribution for the 2D waveguide. The symmetry of the guide is used to reduce the computational time. The lateral position has been curtailed at 3 microns to give a better perspective for the profile.

\section{TEMPORAL DYNAMICS}

The two-dimensional field calculation is incorporated into the carrier density and photon rate equations through the optical confinement factor. For a device of length $250 \mu \mathrm{m}$ and $1.2 \mu \mathrm{m}$ stripe width, self-pulsation has been achieved as seen from the front facet output energy shown in Fig.4. The spreading of the field profile into the absorption sections is predominantly dependent on the carrier density in the active regions under the stripe. Fig. 5 illustrates that as the carrier density increases so too does the full-width-at half-maximum (FWHM) of the field within the active region. This occurs as a result of a reduction in the active later refractive index with increasing carrier density. 


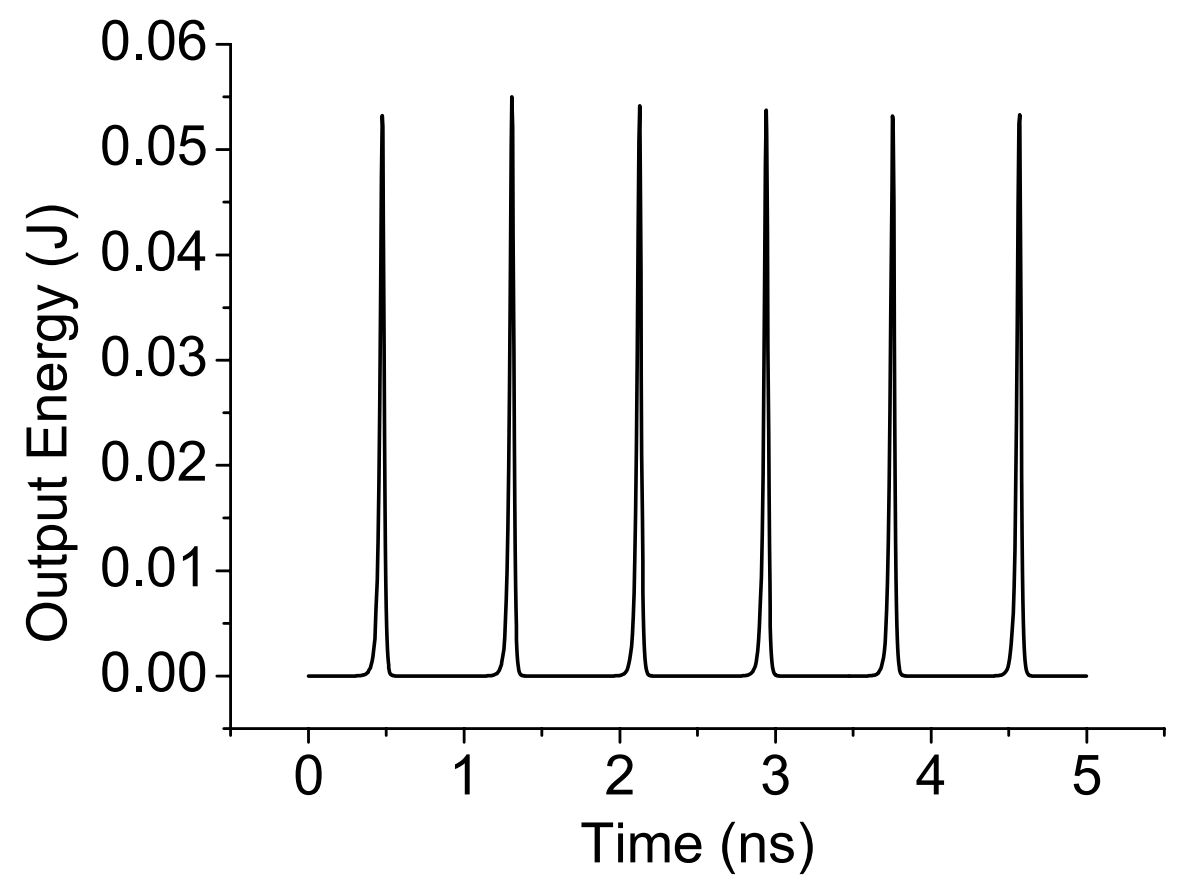

Figure 4. Temporal evolution of the output energy emitted from the front facet of the laser. The bias current was set at $30 \mathrm{~mA}$ and the self-pulsation frequency is $2.23 \mathrm{GHz}$.

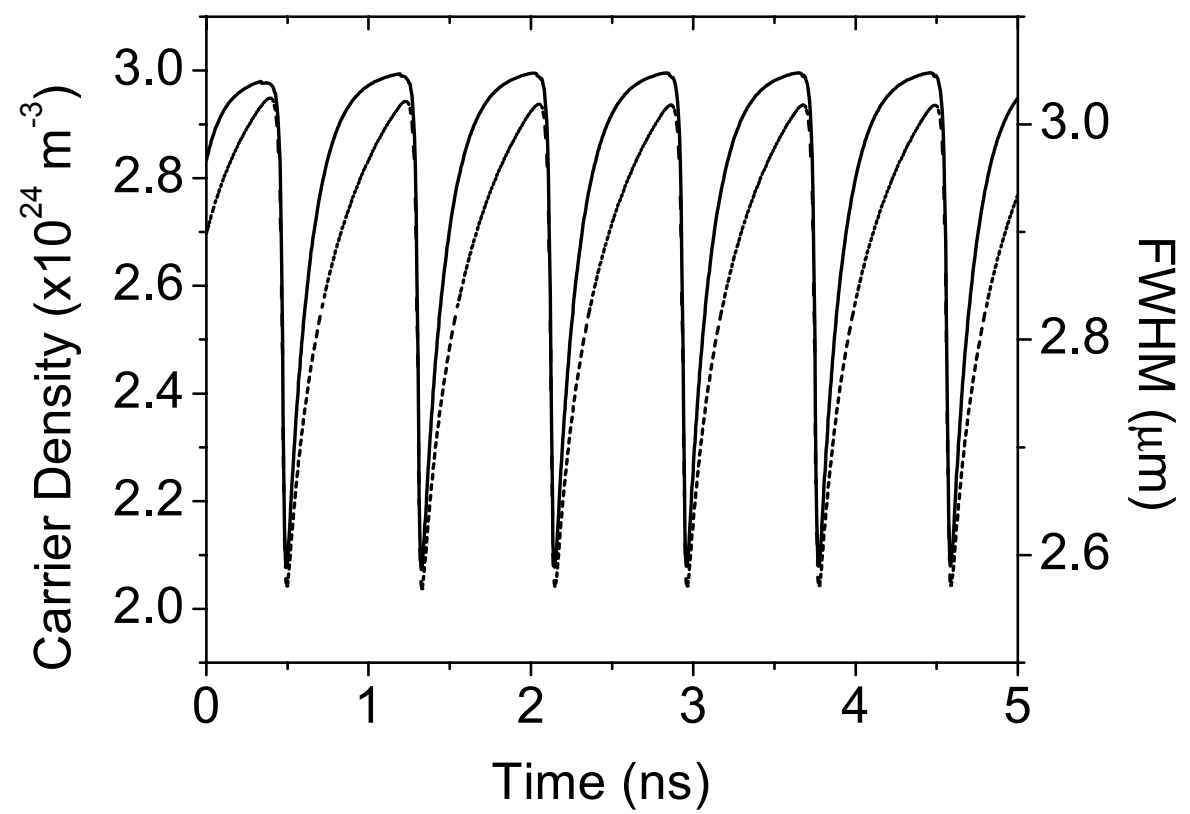

Figure 5 Variation of the FWHM (full line) of the optical field and the carrier density (dashed line) of the centermost section of the device. 
As the pulse is emitted, the loss of carriers due to stimulated emission results in a contraction of the FWHM, a term known as self-focusing. This focusing and defocusing of the electric field is crucial to the self-pulsating mechanism. Another key parameter that is correlated to the peak carrier density is the lasing wavelength. Apart from the case of spatial hole burning in which there is a depletion in the carrier density profile directly under the stripe due to the increased stimulated emission in this region, the central peak carrier density can normally be represented by the carrier density in the center-most section. The variation of the wavelength is shown in Fig. 6 Since the wavelength shift between the maximum and minimum carrier density value is just $0.55 \mathrm{~nm}$, the effect that this has on the optical field profile is quite small. The wavelength decreases with higher carrier density due to the shift in the gain peak to higher energies.

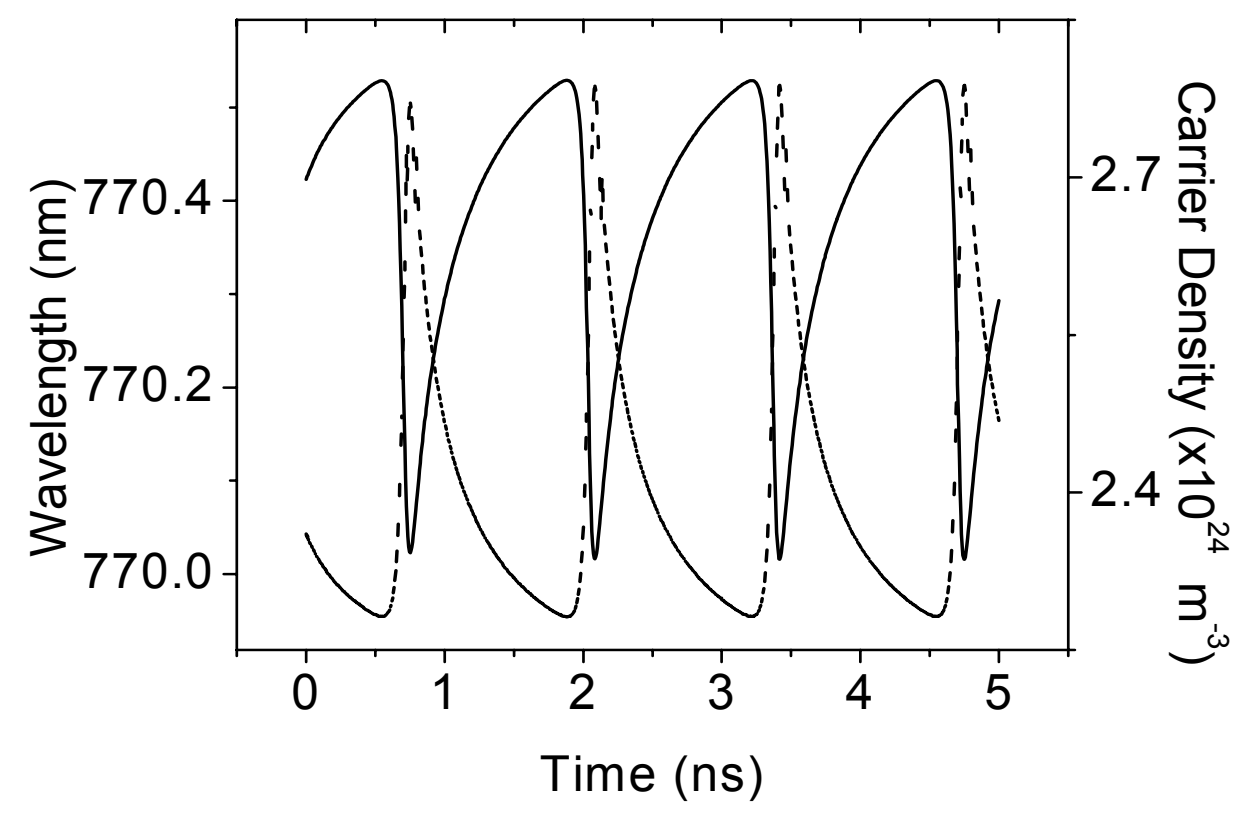

Figure 6 Variation in wavelength (dashed line) and peak (centre- most section) carrier density (full line) with time. The bias current was set at $27 \mathrm{~mA}$.

Using the 2D model, it is possible to make a comparison between the modal gain as calculated from the imaginary component of the longitudinal propagation constant $\operatorname{Im}\left(\beta_{\mathrm{z}}\right)$, with the sum of the product of the material gain and the confinement factor in each section.

$$
\begin{gathered}
G=2 x \operatorname{Im}\left(\beta_{z}\right) \\
G=\sum_{i=1}^{N_{y}} \Gamma(i) g(i)
\end{gathered}
$$

This calculation also serves as a means of testing the validity of the eigenvalues calculated from the Arnoldi method. Figure 7 shows that there is a close agreement between the modal gain calculated from the imaginary component of the longitudinal propagation constant, depicted here in the dashed line, and the modal gain calculated from the sum of the products of the material gain and confinement factor in each section, shown in the full line. It is clear that the agreement is excellent and that our model is very efficient at calculating the appropriate eigenvalues. 


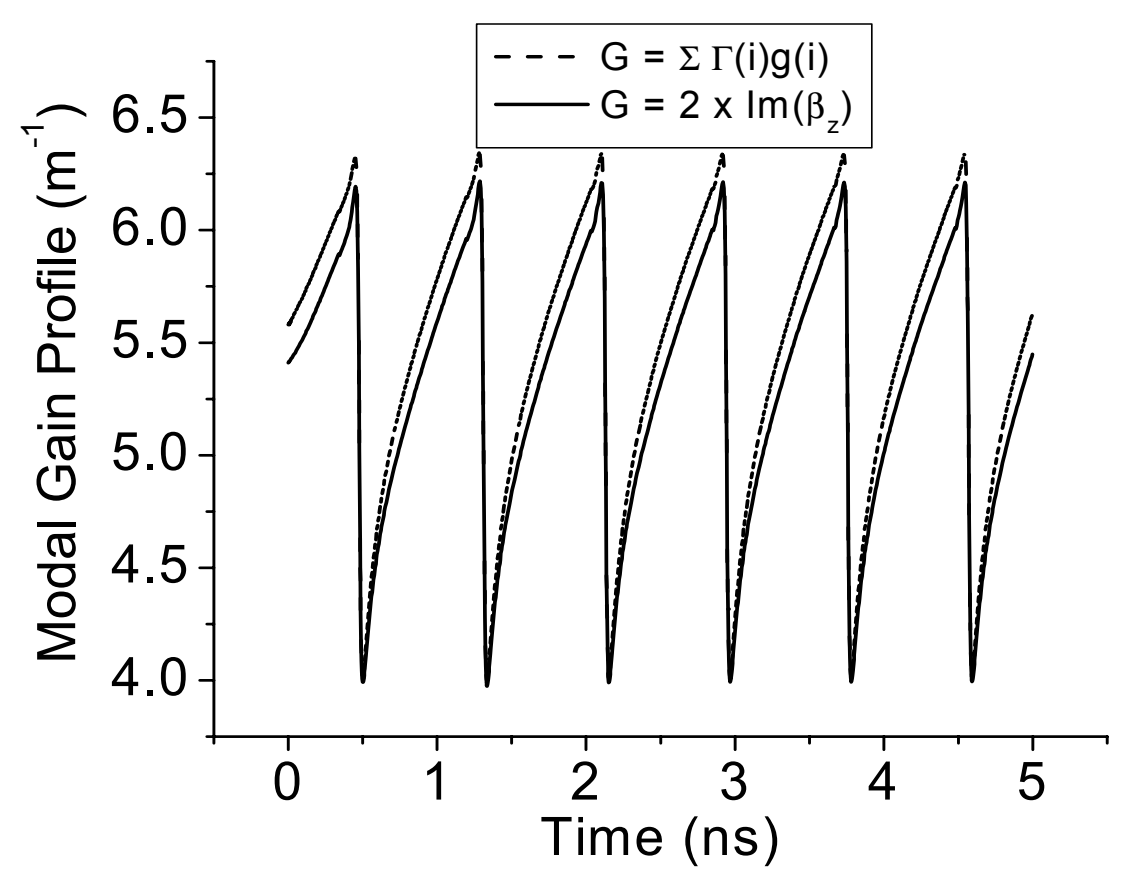

Figure 7 Comparison of the modal gain calculated from the imaginary component of the propagation constant (full line) and the sum of the material gain and confinement factor in each section (dashed line).

\section{DISCUSSION AND CONCLUSIONS}

The fundamental limitations of the use of the effective index method for weakly guided waveguides are clear from Fig.2. Although the lateral waveguide is indeed much weaker than the strong transverse guide, thereby allowing the use of the effective index model, it is important to explore the realm over which the effective index can be used. The reduction of the effective index step by the addition of carrier is a well known effect and if sufficient carriers are introduced, the guide will move from guiding into anti-guiding. In this anti-guiding regime, we find that modes can still be calculated as shown in Fig. 2 but they no longer properly represent the modes of the waveguide. For our studies here, we used a bulk AlGaAs region with Al content of 0.14 . The blocking and cladding layer Al concentrations were 0.55 and 0.50 respectively. For the AlGaAs system, the effective steps in the refractive index profiles are in the region of 3-5 x $10^{-3}$, with a $\frac{d n}{d N}=-1.2 \times 10^{-26} \mathrm{~m}^{-3}$, at carrier densities about $1 \times 10^{24} \mathrm{~m}^{-3}$, which are around threshold values, the guide is at best weakly guiding and so care must be exercised with the use of the effective index model for this material system at least. A more detailed paper describing the usefulness of the effective index model will be published elsewhere ${ }^{10}$.

The breakdown of the effective index method for weakly complex index guided structures prompted the development of a 2D model. Firstly, the waveguide was solved accurately using a non-uniform finite difference grid availing of the symmetry of the waveguide. Secondly, the resulting non-hermitian sparse matrix was solved using a method based on the implicitly restarted Arnoldi method. The accurate determination of the optical modes was shown through the agreement of the modal gain determination based on the imaginary component of the longitudinal propagation constant and its determination as the sum of product of the material gain and confinement factor in each lateral section. 
Incorporating this method into the laser diode model allowed us to study the self-pulsation dynamics. We find that the laser dynamics as shown in Fig.4 are very similar to those found in self-pulsating devices with 1-2 ps pulses and repetition rates of about $2 \mathrm{GHz}$. This in turn shows the usefulness of the model in a predictive mode for the design of self-pulsating lasers. It should be possible to design a diode with the AlGaAs system with specific wavelength and GHz pulse repetition rates.

In the future we plan a study of the laser dynamics above threshold to see if we can accurately model experimental results that have been obtained based on the AlGaAs system.

\section{REFERENCES}

1. R. Brouwer, C. Velzel and B. Yeh, "Lateral modes and self-oscillations in narrow-stripe double heterostructure GaAlAs injection lasers" IEEE J. Quantum Electron 17, 694-701, 1981.

2. J. Van Der Ziel, "Self-focussing effects in pulsating $\mathrm{Al}_{\mathrm{x}} \mathrm{Ga}_{1-\mathrm{x}} \mathrm{As}$ double heterostructure lasers" IEEE J. Quant. Electron. 17, 60-68, 1981.

3. H. Summers and P. Rees, "High temperature operation of $650 \mathrm{~nm}$ wavelength AlGaInP self-pulsating diode lasers" IEEE Photonics Technol. Lett. 10, 1217-1219, 1998.

4. D.R. Jones, P. Rees and I. Pierce, "Optimization of InGaN narrow stripe self-pulsating laser diodes" Conference on Lasers and Electro-Optics Europe - Technical Digest, CWF98, p 236, 2000.

5. M. Yamada, "A theoretical analysis of self-sustained pulsation phenomena in narrow-stripe semiconductor lasers" IEEE J. Quant. Electron. 29, 1330-1336, 1993.

6. J. Manning, R Olshansky and C Bing Su "Carrier-induced index change in AlGaAs and $1.3 \mu \mathrm{m}$ InGaAsP diode lasers", IEEE J. Quant. Electron. 19, 1525-1530, 1983.

7. E. Anderson, Z. Bai, C. Bishof, S. Blackford, J. Demmel, J. Dongarra, J.Du Croz, A. Greenbaum, S. Hammarling, A. McKenney, D. Sorensen, "Lapack Users Guide", Third Edition; Society for Industrial and Applied Mathematics 1999.

8. D.C. Sorensen. "Implicit application of polynomial filters in a k-step Arnoldi method." SIAM J. Matrix Anal. App., 13, 357-385, 1992.

9.R.B. Lehoucq, D.C. Sorensen, C.Yang. "ARPACK Users Guide: Solution of Large Scale Eigenvalue Problems with Implicitly Restarted Arnoldi methods" SIAM Publications, Philidelphia, PA, 1998.

10. C. O'Brien, W-H Seo, H. Thornquist, and J.F. Donegan, (unpublished). 